\title{
Carvedilol Protects against Doxorubicin-Induced Mitochondrial Cardiomyopathy
}

\author{
D. L. Santos,* A. J. M. Moreno, † R. L. Leino, $\S$ M. K. Froberg, $\S^{\prime} \|$ and K. B. Wallace $\S^{\S ! 1}$ \\ *University of Tras-Os-Montes and Alto Douro, Vila Real, $\dagger$ Department of Zoology and Center for Neurosciences and Cell Biology, University of Coimbra, \\ Coimbra, Portugal; and $\$$ Department of Anatomy and Cell Biology, \|Department of Pathology, and ${ }^{\mathbb{I}}$ Department of Biochemistry \\ and Molecular Biology, §University of Minnesota, Duluth, Minnesota 55812-2487
}

Received April 9, 2002; accepted September 27, 2002

C arvedilol Protects against D oxorubicin-Induced Mitochondrial C ardiomyopathy. Santos, D. L., Moreno, A. J. M., Leino, R. L., Froberg, M. K., and Wallace, K. B. (2002). Toxicol. Appl. Pharmacol. 185, 218-227.

Several cytopathic mechanisms have been suggested to mediate the dose-limiting cumulative and irreversible cardiomyopathy caused by doxorubicin. Recent evidence indicates that oxidative stress and mitochondrial dysfunction are key factors in the pathogenic process. The objective of this investigation was to test the hypothesis that carvedilol, a nonselective $\beta$-adrenergic receptor antagonist with potent antioxidant properties, protects against the cardiac and hepatic mitochondrial bioenergetic dysfunction associated with subchronic doxorubicin toxicity. Heart and liver mitochondria were isolated from rats treated for 7 weeks with doxorubicin (2 $\mathrm{mg} / \mathrm{kg} \mathrm{sc} /$ week), carvedilol (1 $\mathrm{mg} / \mathrm{kg}$ ip/week), or the combination of the two drugs. Heart mitochondria isolated from doxorubicin-treated rats exhibited depressed rates for state 3 respiration (336 \pm 26 versus $425 \pm 53$ natom $0 / \mathrm{min} / \mathrm{mg}$ protein) and a lower respiratory control ratio $(R C R)(4.3 \pm 0.6$ versus $5.8 \pm 0.4)$ compared with cardiac mitochondria isolated from saline-treated rats. Mitochondrial calcium-loading capacity and the activity of NADH-dehydrogenase were also suppressed in cardiac mitochondria from doxorubicin-treated rats. Doxorubicin treatment also caused a decrease in RCR for liver mitochondria ( $3.9 \pm 0.9$ versus $5.6 \pm 0.7$ for control rats) and inhibition of hepatic cytochrome oxidase activity. Coadministration of carvedilol decreased the extent of cellular vacuolization in cardiac myocytes and prevented the inhibitory effect of doxorubicin on mitochondrial respiration in both heart and liver. Carvedilol also prevented the decrease in mitochondrial $\mathrm{Ca}^{2+}$ loading capacity and the inhibition of the respiratory complexes of heart mitochondria caused by doxorubicin. Carvedilol by itself did not affect any of the parameters measured for heart or liver mitochondria. It is concluded that this protection by carvedilol against both the structural and functional cardiac tissue damage may afford significant clinical advantage in minimizing the dose-limiting mitochondrial dysfunction and car-

\footnotetext{
${ }^{1}$ To whom correspondence should be addressed at University of Minnesota School of Medicine, Department of Biochemistry and Molecular Biology, 10 University Drive, Duluth, MN. Fax: (218) 726-8014; E-mail: kwallace@ d.umn.edu.
}

diomyopathy that accompanies long-term doxorubicin therapy in cancer patients. $\odot 2002$ Elsevier Science (USA)

Key Words: adriamycin; adrenergic; mitochondria; toxicity.

Doxorubicin (DXR; Adriamycin) is a potent and broadspectrum antineoplastic agent that is prescribed for the treatment of a variety of cancers including leukemias and solid tumors. The cytostatic effect of DXR is attributed to intercalation of the planar anthracycline ring structure into the double helix of nuclear DNA to interfere with DNA replication and transcription, especially in rapidly dividing cells. Unfortunately, the therapeutic success is limited by the development of a dose-dependent and irreversible cardiac toxicity that typically presents as a dilated cardiomyopathy leading to congestive heart failure (Lefrak et al., 1973). Because myocardial cells are mitotically fixed, DXR cardiotoxicity is thought to be manifested via mechanisms other than its cytostatic effect (Olson and Mushlin, 1990; Singal et al., 1997; Singal and Iliskovic, 1998).

There is considerable evidence demonstrating that mitochondria are principal targets in the development of DXRinduced cardiomyopathy (Olson and Mushlin, 1990; Singal et al., 1997; Singal and Iliskovic, 1998). Cytofluorescence microscopy of heart tissue reveals nuclei and mitochondria as exclusive sites of DXR localization (Nicolay et al., 1986) and early stages of DXR cardiomyopathy are characterized by changes in both the morphology and function of heart mitochondria (Ferrero et al., 1976; Mailer and Petering, 1976; Bachmann and Zbinden, 1979; Bachmann et al., 1987), including interference with mitochondrial calcium homeostasis at subclinical cumulative doses (Solem and Wallace, 1993; Solem et al., 1994; Zhou et al., 2000). There is growing evidence that correlates the onset and severity of DXR cardiotoxicity with disturbance in heart mitochondrial function and bioenergetics (Mailer and Petering, 1976; Wallace et al., 1997).

The biochemical mechanism underlying this mitochondrial cardiotoxicity caused by DXR has not been fully elucidated. 
The weight of evidence indicates that DXR cardiotoxicity is mediated by oxygen free radicals (Olson et al., 1981; Powis, 1989; Olson and Mushlin, 1990; Singal et al., 1997, 2000; Singal and Iliskovic, 1998) and that the mitochondrial inner membrane is the primary site of free radical generation and tissue damage (Doroshow, 1983; Davies and Doroshow, 1986; Praet et al., 1988). Doxorubicin undergoes a futile redox cycling on Complex I of the mitochondrial respiratory chain to liberate highly reactive oxygen free radicals. Associated with this is the oxidative modification of a specific calcium-conducting channel leading to induction of the mitochondrial permeability transition, interference with mitochondrial calcium homeostasis, and the disruption of calcium-dependent mitochondrial bioenergetics (Ferrero et al., 1976; Solem and Wallace, 1993; Solem et al., 1994; Zhou et al., 2000). In view of the implication of oxygen free radicals in the pathogenic process, one of the approaches to minimize DXR cardiotoxicity has been through the use of free radical scavengers and other antioxidants (Praet et al., 1988; Siveski-Iliskovic et al., 1995; Singal et al., 1997). However, these attempts have met with mixed success.

Carvedilol (Kredex, Coreg) (1-[carbazolyl-(4)-oxy]-3-[(2methoxyphenoxyethyl) amino]-propanol-(2)), is a novel adrenergic antagonist that competitively blocks $\beta_{1}$ and $\beta_{2}$ vascular receptors, primarily through a selective $\alpha_{1}$-blockade (Ruffolo et al., 1991). The drug has been introduced for the treatment of mild to moderate hypertension and has since been demonstrated to benefit the management of several cardiomyopathies, including ischemia-reperfusion and doxorubicin-induced cardiomyopathy (Feuerstein et al., 1992, 1997; Matsui et al., 1999). For example, carvedilol increases left ventricular ejection fraction with doxorubicin cardiomyopathy (Matsui et al., 1999), but this may be more of a vascular effect than an effect of carvedilol directly on the myocardium itself.

The mechanisms underlying this cardioprotective effect are not fully understood. However, several reports suggest that cardioprotection may be afforded through the potent antioxidant activity of carvedilol, which is not shared by all $\beta$-adrenergic antagonists (Feuerstein et al., 1992; Yue et al., 1992a,b; Kramer and Weglicki, 1996; Tadolini and Franconi, 1998). Carvedilol has been shown to scavenge oxygen free radicals (Yue et al., 1994) and to inhibit lipid peroxidation in biological systems (Yue et al., 1992a,b; Kramer and Weglicki, 1996). Moreover, the antioxidant protection of carvedilol occurs through a chain-breaking mechanism in postischemic rat hearts (Kramer and Weglicki, 1996). The capacity of carvedilol to inhibit lipid peroxidation is much greater than that of other $\beta$-adrenergic blocking agents, such as propranolol (Yue et al., 1992a,b).

The purpose of this investigation was to assess the potential cardioprotectant effect afforded by carvedilol against the free radical-mediated cardiomyopathy and mitochondrial dysfunction that accompanies long-term treatment of rats with clinically relevant cumulative doses of doxorubicin.

\section{EXPERIMENTAL PROCEDURE}

Chemicals. Reagents used in the experiments were as follows: tetraphenylphosphonium chloride (TPPCl) (Aldrich Chemical Co., Milwaukee, WI); ultrapure sucrose (ICN Biomedicals, Aurora, $\mathrm{OH}$ ); calcium chloride, magnesium chloride (Fisher Scientific Company, Fair Lawn, NJ); potassium chloride (EM Science, Gibbstown, NJ); potassium phosphate (J. T. Baker, Phillisburg, $\mathrm{NJ}$ ); arsenazo III (Calbiochem Corp., LaJolla, CA); cyclosporine A (CyA; a generous gift from Sandoz Pharmaceutical Corp., East Hanover, NJ); protease subtilopeptidase A type VIII, EGTA, HEPES, L-glutamic acid, and L-malic acid (Sigma Chemical Company, St Louis, MO). Doxorubicin-HCl (adriamycin) was purchased from Pharmacia \& Upjohn (Milan, Italy). Carvedilol was obtained from Boehringer Mannheim and used in DMSO solution. All reagents were sodium free and reagent grade and were of the highest quality available. Buffers were prepared using water passed through a Millipore Milli Q water purification system (Millipore Corp., Bedford, MA) and the $\mathrm{pH}$ was adjusted to $\mathrm{pH} 7.4$ with $\mathrm{KOH}$ and $\mathrm{HCl}$.

Animals. Adult male Sprague-Dawley rats, purchased from Harlan Sprague-Dawley (Madison, WI) were maintained in a climate-controlled $\left(21^{\circ} \mathrm{C}\right.$, 12-h light cycle), AAALAC-accredited animal care facility with free access to food (Purina Rat Chow, Ralston Purina, Richmond, IN) and tap water. All animals were allowed to acclimate for at least 5 days prior to the first injection. Rats were divided randomly into four treatment groups of six animals each. Each animal received seven weekly injections of doxorubicin $(2 \mathrm{mg} / \mathrm{kg} \mathrm{sc})$, carvedilol ( $1 \mathrm{mg} / \mathrm{kg}$ ip), or saline. Injection sites were rotated to minimize local tissue irritation and injury. Rats were euthanized with $\mathrm{CO}_{2}$ followed by decapitation in the 8 th week ( 1 week after the last of seven weekly injections).

Preparation of heart and liver mitochondria. Heart and liver mitochondria were prepared by conventional methods of differential centrifugation (Solem and Wallace, 1993; Henry et al., 1995). The heart was immediately excised and minced in ice-cold medium containing $250 \mathrm{mM}$ sucrose, $1 \mathrm{mM}$ EGTA, and $5 \mathrm{mM}$ Hepes-KOH, pH 7.4. The minced blood-free tissue was rinsed and suspended in $5 \mathrm{ml}$ of fresh medium and then homogenized with a tightly fitted Potter-Elvehjen homogenizer and a Teflon pestle. Protease (1 mg subtilopeptidase A type VIII prepared in $1 \mathrm{ml}$ of isolation medium) was added and the suspension was rehomogenized for an additional $30 \mathrm{~s}$. The digested homogenate was then immediately diluted with $30 \mathrm{ml}$ medium followed by another $30 \mathrm{~s}$ homogenization. The homogenate was divided into two 50-ml tubes and centrifuged at $11,000 \mathrm{~g}$ for $10 \mathrm{~min}$ at $4^{\circ} \mathrm{C}$. The supernatant was decanted and the pellet, devoid of protease, was gently homogenized to its original volume with isolation medium and a loose-fitting homogenizer. The suspension was centrifuged at $900 \mathrm{~g}$ for $10 \mathrm{~min}$ and the low-speed supernatant was filtered through two layers of cheesecloth and then centrifuged at $9000 \mathrm{~g}$ for $10 \mathrm{~min}$ to pellet the mitochondrial membranes. The high-speed supernatant was discarded and the pellet was gently resuspended and repelleted at $9000 \mathrm{~g}$ for $10 \mathrm{~min}$. EGTA was omitted from the final washing medium. The final mitochondrial pellet was resuspended in isolation medium (without EGTA) at a final concentration of $20-40 \mathrm{mg}$ protein $/ \mathrm{ml}$.

Liver was excised and minced in an ice-cold isolation medium containing $250 \mathrm{mM}$ sucrose, $1 \mathrm{mM}$ EGTA, and $5 \mathrm{mM}$ Hepes-KOH, $\mathrm{pH}$ 7.4. The minced tissue was rinsed twice with isolation medium to remove blood and other debris. The tissue was then homogenized with a tightly fitted Potter-Elvehjen homogenizer and centrifuged at $900 \mathrm{~g}$ for $10 \mathrm{~min}$. The low-speed supernatant was transferred to new centrifuge tubes and subjected to centrifugation at $9000 \mathrm{~g}$ for $10 \mathrm{~min}$. The mitochondrial pellet was washed once and resuspended in isolation medium (without EGTA) to a final concentration of $60-80 \mathrm{mg}$ protein/ml.

All isolation procedures were performed at $0-4^{\circ} \mathrm{C}$. The mitochondrial suspensions were used within 4 (heart mitochondria) or $6 \mathrm{~h}$ (liver mitochondria). Mitochondrial suspensions were maintained on ice $\left(0-4^{\circ} \mathrm{C}\right)$ throughout this period. Isolation procedures yielded well-coupled mitochondria: the respiratory control ratio (RCR) of the isolated mitochondria varied from five to eight (with malate-glutamate as substrate) or three to four (with succinate as substrate), as determined according to the method of Estabrook (1967). Mito- 
chondrial protein was estimated according to the Bradford method (Bradford, 1976) using bovine serum albumin as standard.

Oxygen consumption assays. Oxygen consumption was measured polarographically, at $25^{\circ} \mathrm{C}$, using a YSI Biological Oxygen Monitor (Model 530, Yellow Springs Instrument, Yellow Springs, $\mathrm{OH}$ ) and a Clark-type oxygen electrode connected to a chart recorder (Kipp \& Zonen, model BD 112). Reactions were conducted in a 1-ml closed thermostatted and magnetically stirred glass vessel containing $0.5 \mathrm{mg}$ of mitochondrial protein in a respiration buffer of $130 \mathrm{mM}$ sucrose, $50 \mathrm{mM} \mathrm{KCl}, 2.5 \mathrm{mM} \mathrm{KH}_{2} \mathrm{PO}_{4}$, and $5 \mathrm{mM}$ Hepes, $\mathrm{pH}$ 7.4. After a 1-min equilibration period, mitochondrial respiration was initiated by adding glutamate and malate to a final concentration of $1 \mathrm{mM}$ each. State 3 respiration was measured after adding $0.25 \mathrm{mM}$ ADP; state 4 was measured as the rate of oxygen consumption in the absence of ADP. The RCR (state 3/state 4 rate) and the ADP/O ratio were calculated as described by Estabrook (1967), using 474 ngatom $\mathrm{O} / \mathrm{ml}$ as the value for the solubility of oxygen at $25^{\circ} \mathrm{C}$ in doubly distilled water (Kamo et al., 1979). The concentration of the stock solution of ADP was confirmed spectrophotometrically at 260 $\mathrm{nm}$ using a molecular extinction coefficient of $16 \mathrm{~cm}^{-1} \mathrm{M}^{-1}$.

Mitochondrial membrane potential measurements. Mitochondrial membrane potential $(\Delta \Psi)$ was estimated by monitoring the accumulation and release of the lipophilic cation tetraphenylphosphonium $\left(\mathrm{TPP}^{+}\right)$with a $\mathrm{TPP}^{+}$sensitive electrode prepared according to the procedure described by Kamo (Kamo et al., 1979). A minielectrode was constructed with a poly(vinyl chloride) tube sealed with a poly(vinyl chloride)-based membrane containing tetraphenylboron as a cation exchanger and filled with a $10-\mathrm{mM} \mathrm{TPP}^{+}$solution. The reference electrode was an $\mathrm{Ag} / \mathrm{AgCl}$-saturated electrode. Both the $\mathrm{TPP}^{+}$electrode and the reference electrodes were connected to a $\mathrm{pH}$ meter and inserted into a thermostatted glass chamber. The signals were fed to a dualtrace potentiometric recorder (Kipp \& Zonen, model BD 112). Mitochondria were incubated in the reaction medium $(130 \mathrm{mM}$ sucrose, $50 \mathrm{mM} \mathrm{KCl}, 2.5 \mathrm{mM}$ $\mathrm{KH}_{2} \mathrm{PO}_{4}$, and $5 \mathrm{mM}$ Hepes, pH 7.4) supplemented with $1 \mu \mathrm{M} \mathrm{TPP}{ }^{+}$. This $\mathrm{TPP}^{+}$concentration was chosen to achieve high sensitivity and to avoid possible toxic effects on mitochondria. The transmembrane electric potential was estimated from the following equation $\left(\right.$ at $25^{\circ} \mathrm{C}$ ) assuming that the distribution of $\mathrm{TPP}^{+}$between mitochondria and the medium follows the Nernst equation, as previously described:

$$
\left.\Delta \Psi(\mathrm{mV})=59 \log (\mathrm{v} / V)-59 \log \left(10^{\Delta E / 59}\right)-1\right),
$$

where $\mathrm{v}, V$, and $\Delta E$ represent mitochondrial volume, volume of the incubation medium, and the deflection of the electrode potential from the baseline, respectively. No correction was made for the "passive" binding contribution of $\mathrm{TPP}^{+}$to the mitochondrial membranes because the purpose of the experiments was to show relative changes in potential rather than absolute values. When necessary, the electrode membrane was washed with a suspension of protein to remove hydrophobic reagents and maintain the response time and sensitivity to $\mathrm{TPP}^{+}$. Calculations of the transmembrane potential were based on a mitochondrial matrix volume of $1.1 \mu \mathrm{l} / \mathrm{mg}$ protein.

Measurement of calcium transport by mitochondria: $\mathrm{Ca}^{2+}$ loading capacity. The $\mathrm{Ca}^{2+}$-loading capacity of mitochondria was estimated spectrophotometrically at $660 \mathrm{~nm}$ in a Beckman DU-7400 spectrophotometer, according to previously published procedures (Solem and Wallace, 1993; Zhou et al., 2000). The assay medium ( $130 \mathrm{mM}$ sucrose, $50 \mathrm{mM} \mathrm{KCl}, 2.5 \mathrm{mM} \mathrm{KH}_{2} \mathrm{PO}_{4}$, and $5 \mathrm{mM}$ Hepes, $\mathrm{pH}$ 7.4) was supplemented with $100 \mu \mathrm{M}$ Arsenazo III, $2 \mu \mathrm{M}$ rotenone, and $1 \mu \mathrm{g} / \mathrm{ml}$ oligomycin. Mitochondria were suspended at 0.25 $\mathrm{mg} / \mathrm{ml}$ in $2 \mathrm{ml}$ of medium added to a cuvette maintained at $25^{\circ} \mathrm{C}$. After $1 \mathrm{~min}$ of incubation, $\mathrm{Ca}^{2+}$ was added ( $40 \mathrm{nmol}$ for liver mitochondria and $200 \mathrm{nmol}$ for heart mitochondria), and mitochondria were energized with $10 \mathrm{mM}$ succinate. Where indicated, cyclosporin $\mathrm{A}$ and carvedilol were added just before adding $\mathrm{CaCl}_{2}$. None of these reagents interfere with the spectrophotometric analysis.

Enzyme assays. All assays were performed at $25^{\circ} \mathrm{C}$, with a Beckman DU 7400 spectrophotometer. The activities of complexes I and IV were measured as described by Birch-Machin and collaborators (Taylor et al., 1993) with slight modifications. Mitochondrial samples were subjected to three cycles of fast freeze-thaw in hypotonic buffer $(20 \mathrm{mM}$ potassium phosphate buffer, $\mathrm{pH}$ 7.2) before the enzymatic assays. NADH dehydrogenase was quantitated by the rate of NADH oxidation followed at $340 \mathrm{~nm}$. The NADH:ubiquinone oxidoreductase (Complex I) assay medium contained $25 \mathrm{mM}$ potassium phosphate buffer, $\mathrm{pH} 7.2,5 \mathrm{mM} \mathrm{MgCl}, 2.5 \mathrm{mg} / \mathrm{ml}$ bovine serum albumin (fraction $\mathrm{V}), 0.13 \mathrm{mM} \mathrm{NADH}$, and $65 \mu \mathrm{M}$ decylubiquinone, a soluble short-chain analogue of ubiquinone, and supplemented with $2 \mu \mathrm{g} / \mathrm{ml}$ antimycin $\mathrm{A}$ and 2 $\mathrm{mM}$ potassium cyanide to block electron transport downstream from Complex I. After $1 \mathrm{~min}, 0.25 \mathrm{mg}$ mitochondria was added to initiate the reaction and the initial rate of NADH oxidation was monitored at $340 \mathrm{~nm}(\varepsilon=6.81$ $\mathrm{mM}^{-1} \mathrm{~cm}^{-1}$ ) for $1 \mathrm{~min}$. Complex I activity was inhibited by $2 \mu \mathrm{g} / \mathrm{mL}$ of rotenone. Complex IV specific activity was determined as the rate of oxidation of reduced cytochrome $c$ at $550 \mathrm{~nm}$. Ferrocytochrome $c$ was prepared by reducing ferricytochrome $c$ with excess sodium dithionite, followed by Sephadex G-25 chromatography to remove unreacted $\mathrm{Na}_{2} \mathrm{SO}_{4}$ (Trounce et al., 1996). The reaction mixture consisting of $20 \mathrm{mM}$ potassium phosphate buffer, $\mathrm{pH} 7.0,0.45 \mathrm{mM} n$-dodecyl- $\beta$-D-maltoside, and $15 \mu \mathrm{M}$ ferrocytochrome $c$ was incubated at $25^{\circ} \mathrm{C}$ for $1 \mathrm{~min}$, and mitochondria (10 $\mu \mathrm{g}$ protein) was added to initiate the reaction. The reaction was first order with respect to cytochrome $c$. The pseudo first-order rate constant was calculated and the results are presented as $(\mathrm{min} / \mathrm{mg})^{-1}$ of protein.

Statistical analysis. Results are presented as the mean \pm SEM for the number of experiments indicated in the legends to the figures. Statistical evaluation was performed using the two-tailed Student's $t$ test; differences with a value of $p<0.05$ were considered significant.

Light and electron microscopy. Small blocks of cardiac muscle from the midportion of the lateral wall of the left ventricle were fixed in $2.5 \%$ glutaraldehyde-2\% formaldehyde in $0.1 \mathrm{M}$ phosphate buffer, $\mathrm{pH} 7.4$, postfixed in $1 \% \mathrm{OsO}_{4}$ in the same buffer, dehydrated in graded acetone solutions, and embedded in epon-araldite. One-micrometer-thick sections for light microscopy were stained with $0.25 \%$ toluidine blue in $1 \%$ sodium borate (Zhou et al., 2000). Thin sections for electron microscopy were stained with lead citrate and uranyl acetate.

Tissue examination. The heart tissue was examined for histopathological evidence of cardiomyopathy according to the 0-3 scoring method of Billingham et al. (1978) and Bristow et al. (1981); see also Zhou et al. (2000) using coded slides and electron microscopy grids: grade 0 , no change from normal; grade 1, limited number of isolated cells (less than $5 \%$ of total number of cells per block) exhibiting early myofibrillar loss and/or cytoplasmic vacuolation; grade 2, groups of cells (5 to $30 \%$ of total number) exhibiting marked myofibrillar loss and/or cytoplasmic vacuolization; and grade 3, diffuse cell damage ( $>30 \%$ of total number) with the majority of cells exhibiting marked loss of contractile elements, loss of organelles, and mitochondrial and nuclear degeneration. In addition, damaged cardiomyocytes in a $202,500-\mu \mathrm{m}^{2}$ total cross-sectional region of ventricular wall were counted with the aid of an eyepiece grid and light microscope. These counts were used to compute the mean number of myocytes that were damaged per unit area, resulting in the "cell damage score" reported in Table 6 . All slides were scored independently by two examiners, both of whom were blinded to the tissue sample code.

\section{RESULTS}

\section{Body, Heart, and Liver Weight}

Rats received seven weekly injections of saline, DMSO, doxorubicin ( $2 \mathrm{mg} / \mathrm{kg} \mathrm{sc})$ and/or carvedilol ( $1 \mathrm{mg} / \mathrm{kg}$ ip) and then were killed 1 week following the last injection. All of the animals survived the entire 8-week treatment. However, DXRtreated rats weighed $10-15 \%$ less compared to control rats (Table 1). The lower body weight was significant despite obvious thoracic and abdominal ascites, which was not re- 
TABLE 1

R at Body, Heart, and Liver Weights

\begin{tabular}{lccccc}
\hline \multicolumn{1}{c}{ Treatment } & $\begin{array}{c}\text { Body weight } \\
(\mathrm{g})\end{array}$ & $\begin{array}{c}\text { Heart weight } \\
(\mathrm{g})\end{array}$ & $\begin{array}{c}\text { Liver weight } \\
(\mathrm{g})\end{array}$ & $\begin{array}{c}\text { Heart/body ratio } \\
\left(\times 10^{-3}\right)\end{array}$ & $\begin{array}{c}\text { Liver/body ratio } \\
\left(\times 10^{-3}\right)\end{array}$ \\
\hline Saline & $372 \pm 15$ & $1.2 \pm 0.05$ & $13.5 \pm 0.7$ & $3.2 \pm 0.003$ & $36 \pm 0.05$ \\
Doxorubicin & $325 \pm 30^{*}$ & $0.97 \pm 0.09^{*}$ & $18.4 \pm 1.8^{*}$ & $3.0 \pm 0.003$ & $57 \pm 0.06^{*}$ \\
Carvedilol & $371 \pm 18$ & $1.23 \pm 0.08$ & $13.9 \pm 1.1$ & $3.3 \pm 0.004$ & $37 \pm 0.06$ \\
Doxorubicin + carvedilol & $330 \pm 27^{*}$ & $1.0 \pm 0.06^{*}$ & $16.8 \pm 0.9^{*}$ & $3.3 \pm 0.002$ & $51 \pm 0.03 \dagger$ \\
\hline
\end{tabular}

Note. Values are means $\pm \operatorname{SEM}(n=6)$ for animals receiving seven weekly injections of doxorubicin $2 \mathrm{mg} / \mathrm{kg} / \mathrm{week} \mathrm{sc}$ and carvedilol $1 \mathrm{mg} / \mathrm{kg} / \mathrm{week}$ ip.

* Statistically significant compared to control group $(p<0.05)$.

$\dagger$ Statistically significant compared to doxorubicin group $(p<0.05)$.

versed by concomitant treatment with carvedilol. Heart weight was also lower in DXR-treated rats, with or without concomitant carvedilol treatment (Table 1). However, heart/body weight ratio was not altered by any combination of drug treatments. The liver of DXR-treated rats was congested and enlarged due to the ascites, which was not prevented by carvedilol.

\section{Respiration and Oxidative Phosphorylation of Isolated Mitochondria}

The effects of the different drug treatments on state 3 and state 4 respiration, RCR, and coupling of oxidative phosphorylation (ADP/O ratio) of heart and liver mitochondria are presented in Tables 2 and 3, respectively. Chronic DXR administration resulted in compromised respiratory function of cardiac mitochondria (Table 2). Following DXR treatment, state 3 respiration rate was decreased $20 \%$ compared with control $(336 \pm 26$ versus $425 \pm 53, p<0.05)$, whereas state 4 respiration was not altered. Consequently, the RCR of cardiac mitochondria from DXR-treated rats was significantly

TABLE 2

Effects of D oxorubicin Treatment on Oxidative Phosphorylation in Rat Heart Mitochondria

\begin{tabular}{lllll}
\hline & \multicolumn{4}{c}{ Respiration rate (natoms O/min/mg protein) } \\
\cline { 2 - 5 } \multicolumn{1}{c}{ Treatment } & State 3 & State 4 & RCR & ADP/O \\
\hline Saline & $425 \pm 53$ & $74 \pm 11$ & $5.8 \pm 0.4$ & $2.7 \pm 0.2$ \\
Doxorubicin & $336 \pm 26^{*}$ & $79 \pm 11$ & $4.3 \pm 0.6^{*}$ & $3.0 \pm 0.2$ \\
Carvedilol & $417 \pm 55$ & $72 \pm 8$ & $5.8 \pm 0.5$ & $2.6 \pm 0.3$ \\
Doxorubicin + carvedilol & $418 \pm 62 \dagger$ & $70 \pm 13$ & $6.0 \pm 0.5 \dagger$ & $2.7 \pm 0.2$ \\
\hline
\end{tabular}

Note. Data represent the means \pm SEM for cardiac mitochondria isolated from six individual rats receiving weekly injections for 7 consecutive weeks. Oxidative phosphorylation was measured polarographically at $25^{\circ} \mathrm{C}$ in a total volume of $1 \mathrm{ml}$. Respiration medium and other experimental details are given under Experimental Procedure. State 3 and state 4 respiration rates, RCR, and ADP/O were calculated as described under Experimental Procedure.

$* p<0.05$ compared to control.

$\dagger p<0.05$ compared to doxorubicin-treated rats.
(25\%) lower compared to controls $(4.3 \pm 0.6$ and $5.8 \pm 0.4$, respectively, $p<0.05)$. However, the efficiency of ATP synthesis as indicated by ADP/O ratio for cardiac mitochondria was not impaired following DXR treatment. Carvedilol treatment by itself did not alter any of the parameters of mitochondrial respiration. However, when coadministered with DXR, carvedilol prevented the DXR-induced inhibition of state 3 respiration rate and restored the RCR of cardiac mitochondria to control values (Table 2).

The effects of DXR and carvedilol treatment of liver mitochondria are summarized in Table 3. Although neither drug treatment altered either state 3 or state 4 respiration by liver mitochondria, DXR did cause a significant inhibition of RCR, which was prevented by the coadministration of carvedilol ( $3.9 \pm 0.9$ versus $5.4 \pm 0.8$, respectively). As was observed for heart, the $\mathrm{ADP} / \mathrm{O}$ ratio for liver mitochondria was not altered by DXR treatment. Carvedilol by itself did not affect any of the studied mitochondrial respiratory parameters from either liver or heart mitochondria (Tables 2 and 3).

\section{TABLE 3}

Effects of D oxorubicin Treatment on Oxidative Phosphorylation in Rat Liver Mitochondria

\begin{tabular}{lrrrr}
\hline & \multicolumn{4}{c}{ Respiration rate (natoms O/min/mg protein) } \\
\cline { 2 - 5 } \multicolumn{1}{c}{ Treatment } & State 3 & State 4 & RCR & ADP/O \\
\hline Saline & $111 \pm 20$ & $20 \pm 3$ & $5.6 \pm 0.7$ & $2.6 \pm 0.3$ \\
Doxorubicin & $90 \pm 18$ & $24 \pm 4$ & $3.9 \pm 0.9 *$ & $2.8 \pm 0.4$ \\
Carvedilol & $115 \pm 17$ & $22 \pm 2$ & $5.3 \pm 0.7$ & $2.5 \pm 0.3$ \\
Doxorubicin + carvedilol & $101 \pm 28$ & $18 \pm 4$ & $5.4 \pm 0.8 \dagger$ & $2.7 \pm 0.3$ \\
& & & &
\end{tabular}

Note. Data represent the means \pm SEM for cardiac mitochondria isolated from six individual rats receiving weekly injections for 7 consecutive weeks. Oxidative phosphorylation was measured polarographically at $25^{\circ} \mathrm{C}$ in a total volume of $1 \mathrm{ml}$. Respiration medium and other experimental details are given under Experimental Procedure. State 3 and state 4 respiration rates, RCR, and ADP/O were calculated as described under Experimental Procedure.

$* p<0.05$ compared to control.

$\dagger p<0.05$ compared to doxorubicin-treated rats. 

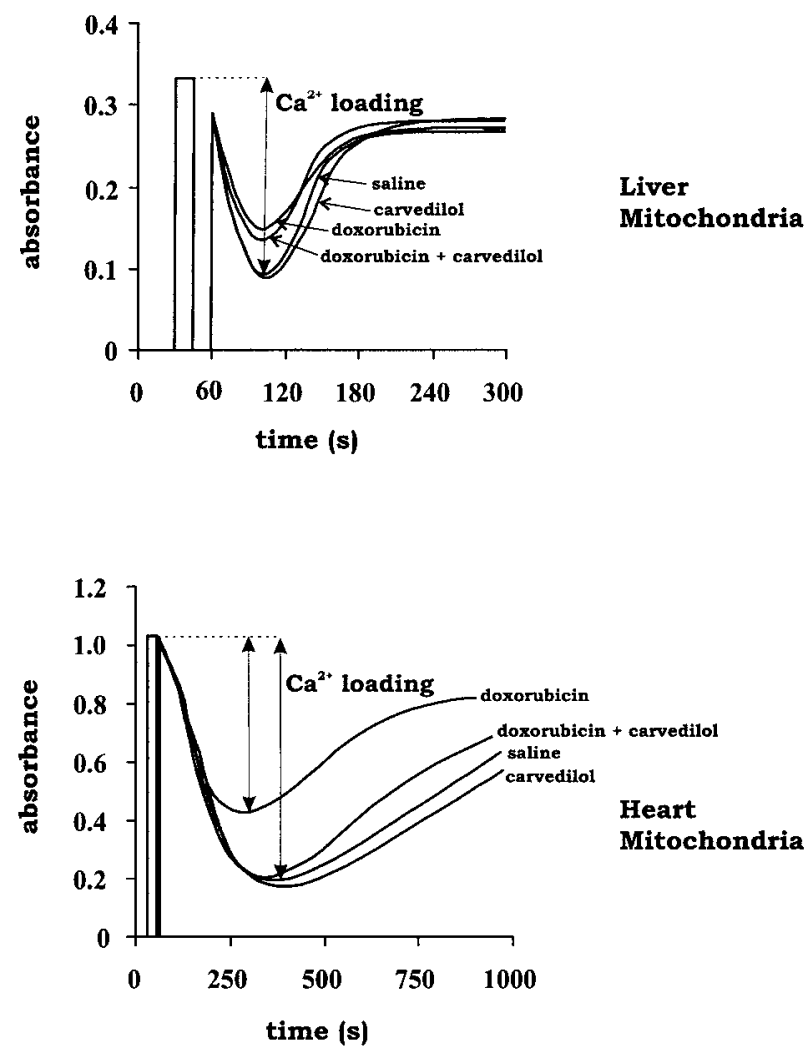

FIG. 1. Quantification of the mitochondrial calcium loading capacity. Freshly isolated rat heart or liver mitochondria were suspended at $0.25 \mathrm{mg}$ protein/ml in a medium containing $130 \mathrm{mM}$ sucrose, $50 \mathrm{mM} \mathrm{KCl}, 2.5 \mathrm{mM}$ $\mathrm{KH}_{2} \mathrm{PO}_{4}$, and $5 \mathrm{mM}$ Hepes ( $\mathrm{pH} 7.4$ ), to which was added $2 \mu \mathrm{M}$ rotenone, 1 $\mu \mathrm{g} / \mathrm{ml}$ oligomycin, $100 \mu \mathrm{M}$ arsenazo III, and 40-200 nmol calcium. The active accumulation of calcium by the mitochondria was initiated by adding 10 $\mathrm{mM}$ succinate and the absorbance monitored continuously for $15 \mathrm{~min}$. The traces are representative of several replications for each type of mitochondrial preparation.

\section{Mitochondrial $\mathrm{Ca}^{2+}$-Loading Capacity}

Figure 1 illustrates the method employed for estimating mitochondrial calcium loading capacity for both heart and liver. Mitochondria were suspended in a medium containing rotenone, oligomycin, calcium, and arsenazo III, which complexes with free calcium to yield a blue color that is quantified by spectrophotometry at $650 \mathrm{~nm}$. Immediately upon addition of succinate to energize the mitochondria, the absorbance of the complex rapidly bleaches as the calcium is taken up into the mitochondria. However, the absorbance subsequently begins to increase as the intramitochondrial calcium concentration increases to the point of triggering the mitochondrial permeability transition, which releases the accumulated calcium back to the extramitochondreal medium to complex once again with the arsenazo dye. Calcium loading capacity is defined as the maximum amount of calcium accumulated by the mitochondrial suspensions before inducing the permeability transition and is expressed as nmol calcium/mg mitochondrial protein.
Performed under comparable conditions, the calcium loading capacity was four- to five-fold greater for cardiac mitochondria than for liver (Table 4). Treatment of rats with DXR, but not carvedilol, caused a significant reduction in the calcium loading capacity of both heart and liver mitochondria. The decrease in calcium loading capacity for liver mitochondria was only $10 \%$, whereas DXR treatment reduced mitochondrial calcium loading capacity by nearly $50 \%$ for heart. Interestingly, although cotreatment with carvedilol protected cardiac mitochondria from DXR-induced decrease in calcium loading capacity, carvedilol did not prevent the effect on liver mitochondria. Conversely, adding cyclosporin A, a potent and specific inhibitor of the mitochondrial permeability transition (Broekemeier et al., 1989), to the reaction medium in vitro restored mitochondrial calcium loading capacity for both heart and liver to values greater than that observed for saline-injected controls. This is consistent with cyclosporin preventing the recycling of calcium across the inner membrane of cardiac mitochondria (Solem and Wallace, 1993; Zhou et al., 2000).

\section{Mitochondrial Electron Chain Enzyme Activities}

A final indication of the effects of DXR and carvedilol treatment in vivo on mitochondrial bioenergetics was to measure the specific activities of Complex I and Complex IV in heart and liver mitochondria from treated rats (Table 5). Subchronic treatment with DXR caused a significant (35\%) inhibition of cardiac mitochondrial Complex I activity, but the activity of Complex IV was unaltered. Contrast this with the effects of DXR in liver, where Complex IV, but not Complex I, was inhibited by treatment with DXR in vivo. In both cases, however, coadministration of carvedilol prevented the inhibitory effect of DXR on mitochondrial respiratory enzyme activity.

\section{Histopathology}

When examined by light and electron microscopy, cardiomyocytes from carvedilol-injected rats exhibited normal mor-

TABLE 4

Heart and Liver Mitochondrial $C \mathrm{a}^{2+}$-Loading $\mathrm{C}$ apacity

\begin{tabular}{llc}
\hline \multicolumn{1}{c}{ Treatment } & $\begin{array}{c}\text { Heart } \\
\text { mitochondria } \\
(\mathrm{nmol} / \mathrm{mg} \text { protein) }\end{array}$ \\
\hline Saline & \multicolumn{1}{c}{$\begin{array}{c}\text { Liver } \\
\text { mitochondria }\end{array}$} \\
Doxorubicin & $695 \pm 145$ & $144 \pm 12$ \\
Carvedilol & $379 \pm 143^{*}$ & $127 \pm 10^{*}$ \\
Doxorubicin/carvedilol & $715 \pm 86$ & $143 \pm 13$ \\
Doxorubicin + cyclosporine A & $696 \pm 60 \dagger$ & $124 \pm 5^{*}$ \\
\hline
\end{tabular}

Note. Values are means $\pm \operatorname{SEM}(n=6)$ for animals receiving seven weekly injections of doxorubicin ( $2 \mathrm{mg} / \mathrm{kg} /$ week sc) and carvedilol ( $1 \mathrm{mg} / \mathrm{kg} /$ week ip).

* Statistically significant compared to control group $(p<0.05)$.

$\dagger$ Statistically significant compared to doxorubicin group $(p<0.05)$. 
TABLE 5

Activities of Mitochondrial Chain Complexes I and IV in Heart and Liver Mitochondria

\begin{tabular}{lccccc} 
& \multicolumn{2}{c}{ Heart } & & \multicolumn{2}{c}{ Liver } \\
\cline { 2 - 3 } \cline { 5 - 6 } \cline { 5 - 5 } Treatment & $\begin{array}{c}\text { Complex I } \\
(\mathrm{nmol} / \mathrm{mg} / \mathrm{min})\end{array}$ & $\begin{array}{c}\text { Complex IV } \\
(\mathrm{k} / \mathrm{mg} / \mathrm{min})\end{array}$ & $\begin{array}{c}\text { Complex I } \\
(\mathrm{nmol} / \mathrm{mg} / \mathrm{min})\end{array}$ & $\begin{array}{c}\text { Complex IV } \\
(\mathrm{k} / \mathrm{mg} / \mathrm{min})\end{array}$ \\
\hline Saline & $7.0 \pm 0.9$ & $264 \pm 24$ & & $0.56 \pm 0.03$ & $162 \pm 10$ \\
$\begin{array}{l}\text { Doxorubicin } \\
\text { Carvedilol }\end{array}$ & $4.5 \pm 0.6^{*}$ & $280 \pm 36$ & & $0.50 \pm 0.09$ & $106 \pm 26^{*}$ \\
$\begin{array}{l}\text { Doxorubicin }+ \\
\quad \text { carvedilol }\end{array}$ & $6.0 \pm 0.9$ & $270 \pm 26$ & & $0.49 \pm 0.05$ & $142 \pm 10$ \\
\hline
\end{tabular}

Note. Enzyme activities were determined as described under Experimental Procedure. Data represent \pm SEM for four independent mitochondrial preparations.

* Statistically significant compared to control, $p<0.05$.

$\dagger$ Statistically significant compared to doxorubicin-treated rats, $p<0.05$.

phology and were indistinguishable from controls (Fig. 2A). However, as reported previously (Zhou et al., 2000), grade I cardiomyopathy occurred in all rats injected with doxorubicin, as illustrated by the appearance of enlarged, swollen mitochondria and vacuoles within the cytoplasm (Figs. 2B and 3A, Table 6). Carvedilol appeared to have a significant cardioprotective effect on cardiac myocytes of rats treated with doxorubicin (Table 6). One of four rats in the doxorubicin + carvedilol treatment group was classified as grade 0 and three of four were classified as grade 1, that is less than $5 \%$ of the myocytes showed damage. Compared to doxorubicin treatment alone, there were fewer damaged cells in the carvedilol + doxorubicin-treated rats, and those cells that were damaged usually contained fewer and smaller vacuoles (Fig. 2C). When examined by electron microscopy, this cardioprotection was apparent by the fewer and less extensively swollen cardiac mitochondria with less distension of the t-tubules and sarcoplasmic reticulum (Fig. 3B).

\section{DISCUSSION}

Several hypotheses have been advanced to explain the doselimiting cumulative and irreversible cardiotoxicity caused by doxorubicin (Singal et al., 1987; Olson and Mushlin, 1990), most of which involve the generation of reactive oxygen species. An emerging hypothesis expands on this concept to implicate free radical-mediated interference with mitochondrial bioenergetics and calcium regulation as being definitive in the pathogenic process (Ferrero et al., 1976; Wallace, 2000). Doxorubicin associates with cardiolipin of the inner mitochondrial membrane, in which is embedded Complex I of the respiratory chain. It is Complex I that catalyzes the futile redox cycling of the doxorubicin to liberate reactive oxygen free radicals (Doroshow, 1983), which in turn alters the thioldependent regulation of the mitochondrial permeability transi- tion pore (Sokolove, 1988; Solem and Wallace, 1993). As a result, doxorubicin stimulates the cyclosporine A-sensitive disruption of mitochondrial calcium homeostasis and bioenergetics (Solem et al., 1994, 1996; Zhou et al., 2000), which among other things leads to interference with calcium-dependent cell signaling pathways (Zhou et al., 2001). Additional indications of oxidative injury to mitochondria include membrane lipid peroxidation (Mimnaugh et al., 1983, 1985), inhibition of respiration and oxidative phosphorylation, decreased mitochondrial ATPase activity, and a net decrease in the redox state of respiratory carriers (Demant and Jensen, 1983; Praet et al., 1984; Praet and Ruysschaert, 1993; Ji and Mitchell, 1994).

In light of the implication of oxidative stress in the pathogenic mechanism, a number of attempts have been made to assess the value of antioxidants in protecting against doxorubicin cardiomyopathy (Praet et al., 1988; Singal et al., 1997). Recent research has revealed three potential therapeutic agents effective in limiting the extent of DXR-induced cardiomyopathy, all of which are based on the antioxidant properties of the candidate compound (Dorr, 1996). Dexrazoxane decreases DXR-induced cardiotoxicity by chelating essential transition metals required for the generation of oxygen free radicals (Seifert et al., 1994; Imondi, 1998; Herman et al., 2000). The fact that dexrazoxane reduces the toxicity but not the antineoplastic activity of DXR is evidence for two distinct mechanisms. Cardioprotection has also been demonstrated for the lipid-lowering drug probucol (Singal et al., 1995, 2000) and the aminothiol amifostine (Herman et al., 2000), both of which express potent oxygen radical scavenging and antioxidant activity in vitro. In both instances, cardioprotection against DXRinduced toxicity is ascribed to the antioxidant properties of the drug (Dorr, 1996).

Carvedilol offers another example of a drug candidate that offers promising protection against cardiac failure (Feuerstein et al., 1992; Kramer and Weglicki, 1996). The drug has been widely prescribed to improve left ventricular function as the result of episodes of ischemia and infarction, neurohumoral mediated cardiac hypertrophy, and dilated cardiomyopathy associated with long-term dialysis (Lahiri, 1996; Tepper, 2001; Jagasia and Shivkumar, 2002; Ponicke et al., 2002; Yaoita et al., 2002). More recently, carvedilol has been shown to improve left ventricular function in rats administered DXR (Matsui et al., 1999). Invariably, the effectiveness of carvedilol has been ascribed to its potent antioxidant properties, which are not shared by all $\beta$-adrenergic antagonist drugs (\#519; Feuerstein et al., 1992, 1997, 1998; Yue et al., 1994; Kramer and Weglicki, 1996; Matsui et al., 1999; Lysko et al., 2000). Although there is substantial evidence to implicate that the cardioprotection is due to the strong antioxidant properties of carvedilol, we can only infer from these previous reports. It remains feasible that cardioprotection was manisfested by the vascular effects of this $\beta$-adrenergic antagonist.

Although considered to be cardioselective, DXR elicits adverse effects in other tissues (e.g., liver) as well. The drug 

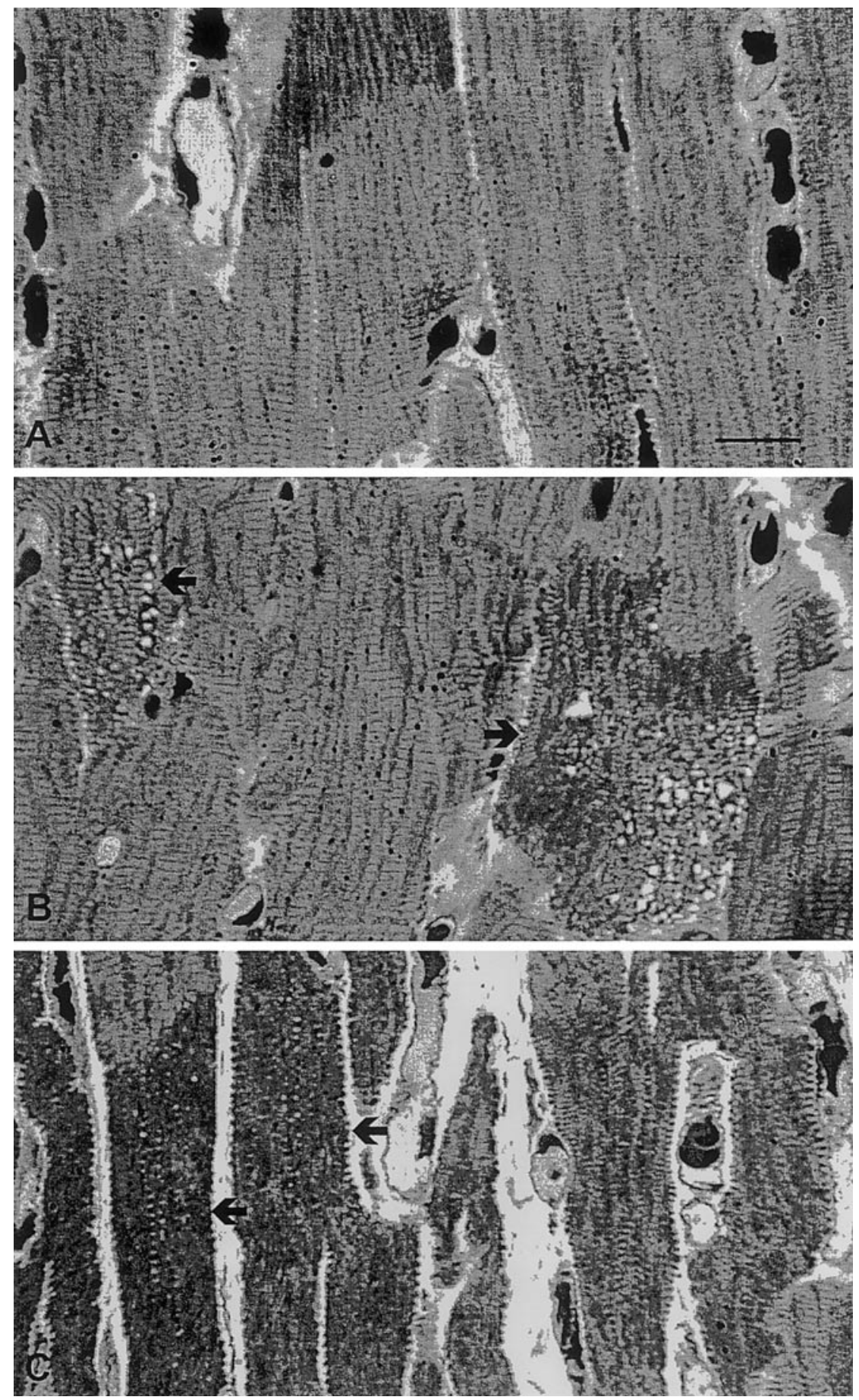

FIG. 2. Light micrographs of cardiac myocytes ( $700 \times$, toluidine blue stain). (A) Control myocytes of normal morphology. (B) Myocytes from doxorubicin-treated rats show extensive vacuolization (arrows). (C) Myocytes from rats treated with doxorubicin plus carvedilol exhibit less extensive vacuolization, with smaller and more sparsely distributed vacuoles compared to B. Bar represents $9 \mu \mathrm{m}(\mathrm{A}-\mathrm{C})$.

distributes throughout the systemic circulation and the cardiotoxicity does not appear to be due to a preferential accumulation in the heart (Yesair et al., 1972). For example, heart and liver mitochondria have comparable capacities to bind adriamycin in vitro (Cheneval et al., 1985). Rather than being a pharmacokinetic phenomenon, the difference in susceptibility may be due to the relatively low levels of superoxide dismutase, catalase, and glutathione peroxidase in cardiac tissue compared to liver (Doroshow et al., 1980) or because of the vastly greater numbers of mitochondria and higher energy 
requirements of the heart (Wallace, 1992, 2000; Wallace et al., 1997). Alternatively, heart mitochondria possess an exogenous NADH dehydrogenase that is not present in liver. This enzyme shuttles unpaired electrons to doxorubicin to generate superoxide free radicals, which have been implicated in the deleterious effects of DXR on heart tissue (Gille and Nohl, 1997). Carvedilol was recently shown to be a potent inhibitor of the external NADH dehydrogenase in heart mitochondria (Oliveira et al., 2000). Accordingly, inhibition of the endogenous NADH dehydrogenase by carvedilol may prevent the enzyme-catalyzed generation of oxygen free radicals, which supposedly mediate the oxidative injury to the cardiac mitochondria. Carvedilol has also been shown to possess potent oxygen free radical scavenging activity (Kramer and Weglicki, 1996), which may contribute to the preservation of cardiac tissue
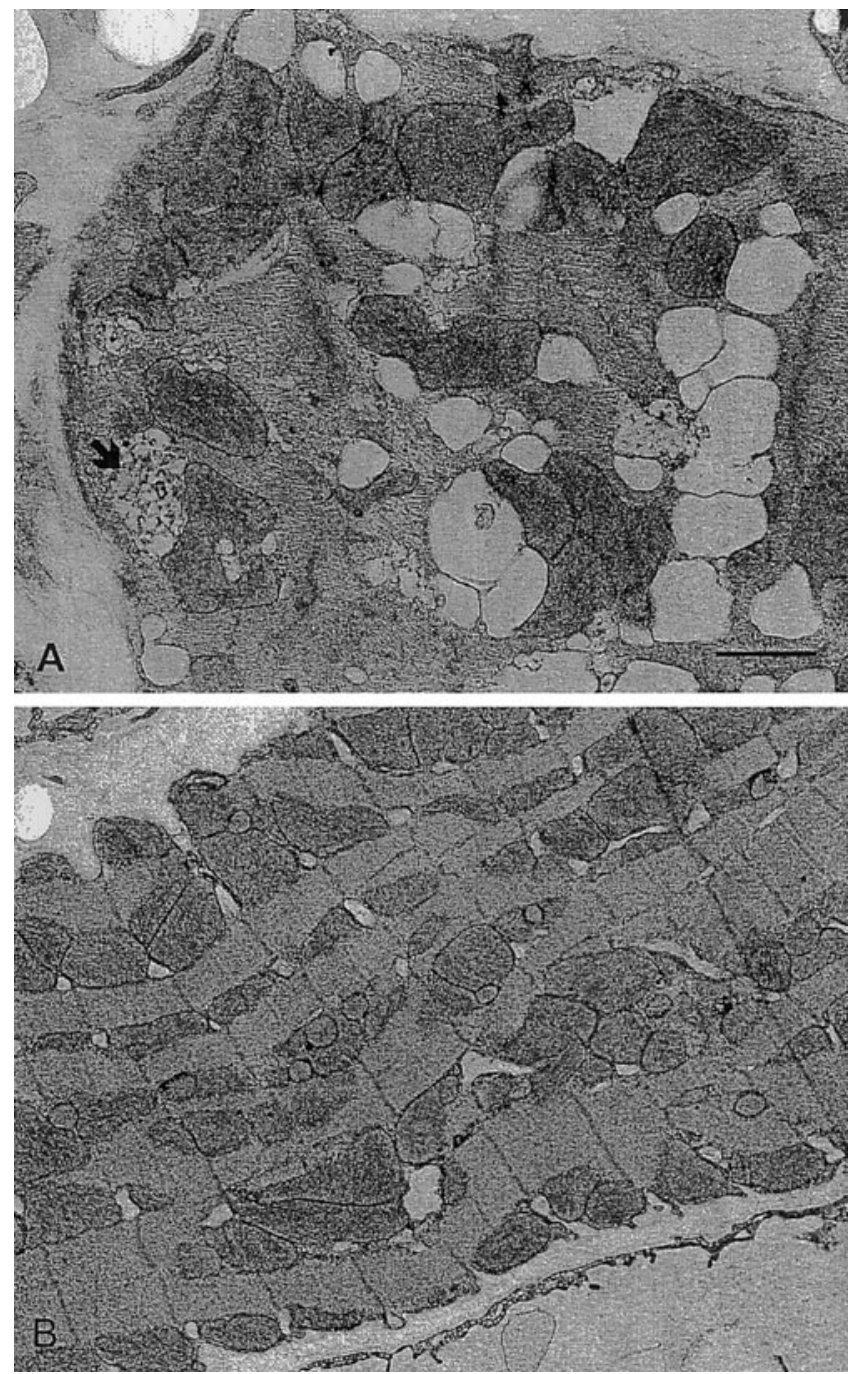

FIG. 3. Representative electron micrographs of cardiac myocytes. (A) Myocytes from doxorubicin treated rats showing large vacuoles and damaged mitochondrial structures (arrows). (B) Myocytes from rats treated with doxorubicin plus carvedilol showing smaller vacuoles with no damage to the mitochondrial structures. Bar represents $1 \mu \mathrm{m}(\mathrm{A})$ and $1.4 \mu \mathrm{m}(\mathrm{B})$.
TABLE 6

Histopathological Grading and Cardiomyocyte Damage in R ats Treated with Doxorubicin and/or C arvedilol

\begin{tabular}{lcc}
\hline \multicolumn{1}{c}{ Treatment } & Mean grade $^{a}$ & Cell damage score $^{b}$ \\
\hline Saline & 0.00 & $0.00 \pm 0.00$ \\
Carvedilol & 0.25 & $0.75 \pm 0.75$ \\
Doxorubicin & 1.00 & $8.75 \pm 1.75$ \\
Doxorubicin + carvedilol & 0.75 & $4.25 \pm 1.18^{*}$ \\
\hline
\end{tabular}

${ }^{a}$ Mean grade is expressed on a scale of $0-3$ as assessed independently by two separate scorers.

${ }^{b}$ Cell damage score represents the mean \pm SEM number of damaged myocytes in a $202,500 \mu \mathrm{m}^{2}$ area.

* Significantly different from doxorubicin treatment, $p<0.05(n=4)$.

structure, mitochondrial bioenergetics, and contractile performance in response to clinically limiting doses of doxorubicin.

Mitochondria isolated from DXR-treated rats exhibited a marked dose-dependent reduction of oxygen consumption and calcium loading capacity, whereas oxidative phosphorylation (ADP/O ratio) remained unchanged. Inhibition of state 3 respiration in cardiac mitochondria isolated from rats treated subchronically with DXR agrees with previous reports concerning the acute toxicity of DXR (Bachmann and Zbinden, 1979). Whether this reflects the inhibition of complex I of the respiratory chain or an induction of the permeability transition and uncoupling of oxidative phosphorylation remain to be determined. Regardless, the drug-dependent decrease in RCR suggests that DXR interferes with the fundamental regulation of oxidative phosphorylation in both cardiac and hepatic mitochondria. The fact that carvedilol prevented the DXR-induced interference with mitochondrial bioenergetics suggests that, regardless of the underlying cause, the protective effect of carvedilol is quite possibly at the level of preserving the redox status of critical mitochondrial elements.

In conclusion, DXR-induced cardiomyopathy is mediated by free radicals generated on the mitochondrial membranes, either by the NADH dehydrogenase or Complex I of the respiratory chain. Regardless of the source, the free radicals oxidize key structural elements to interfere with mitochondrial bioenergetics, which is a root cause for the contractile failure associated with DXR cardiomyopathy. Carvedilol, because of its potent antioxidant properties, offers a new therapeutic strategy, targeted specifically at the myocardium, in limiting the extent of cardiomyopathy associated with cumulative DXR therapy. Carvedilol may be of particular advantage over current therapies in that it belongs to the class of $\beta$-adrenergic antagonists that are widely prescribed as being of essential benefit in the management of cardiogenic disorders, including drug-induced congestive heart failure (Lahiri, 1996; Foody et al., 2002).

\section{ACKNOWLEDGMENTS}

The authors appreciate the expert technical assistance by Juline Smith. This work was supported by NIH Grant HL-58016. 


\section{REFERENCES}

Bachmann, E., Weber E., and Zbinden, G. (1987). Effects of mitoxantrone and doxorubicin on energy metabolism of the rat heart. Cancer Treat. Rep. 71, 361-366.

Bachmann, E., and Zbinden, G. (1979). Effect of doxorubicin and rubidazone on respiratory function and $\mathrm{Ca}^{2+}$ transport in rat heart mitochondria. Toxicol. Lett. 3, 29-34.

Billingham, M. E., Mason, J. W., Bristow, M. R., and Daniels, J. R. (1978). Anthracycline cardiomyopathy monitored by morphologic changes. Cancer Treat. Rep. 62, 865-872.

Bradford, M. M. (1976). A rapid and sensitive method for the quantitation of microgram quantities of protein utilizing the principle of protein-dye binding. Anal. Biochem. 72, 248-254.

Bristow, M. R., Minobe, W. A., Billingham, M. E., Marmor, J. B., Johnson, G. A., Ishimoto, B. M., Sageman, W. S., and Daniels, J. R. (1981). Anthracycline-associated cardiac and renal damage in rabbits: Evidence for mediation by vasoactive substances. Lab. Invest. 45, 157-168.

Broekemeier, K. M., Dempsey, M. E., and Pfeiffer, D. R. (1989). Cyclosporin $\mathrm{A}$ is a potent inhibitor of the inner membrane permeability transition in liver mitochondria. J. Biol. Chem. 264, 7826-7830.

Cheneval, D., Muller, M., Toni, R., Ruetz, S., and Carafoli, E. (1985). Adriamycin as a probe for the transversal distribution of cardiolipin in the inner mitochondrial membrane. J. Biol. Chem. 260, 13003-13007.

Davies, K. J., and Doroshow, J. H. (1986). Redox cycling of anthracyclines by cardiac mitochondria. I. Anthracycline radical formation by NADH dehydrogenase. J. Biol. Chem. 261, 3060-3067.

Demant, E. J., and Jensen, P. K. (1983). Destruction of phospholipids and respiratory-chain activity in pig-heart submitochondrial particles induced by an adriamycin-iron complex. Eur. J. Biochem. 132, 551-556.

Doroshow, J. H. (1983). Anthracycline antibiotic-stimulated superoxide, hydrogen peroxide, and hydroxyl radical production by NADH dehydrogenase. Cancer Res. 43, 4543-4551.

Doroshow, J. H., Locker, G. Y., and Myers, C. E. (1980). Enzymatic defenses of the mouse heart against reactive oxygen metabolites: Alterations produced by doxorubicin. J. Clin. Invest. 65, 128-135.

Dorr, R. T. (1996). Cytoprotective agents for anthracyclines. Semin. Oncol. 23(Suppl. 8), 23-34.

Estabrook, R. W. (1967). Mitochondrial respiratory control and the polarographic measurement of ADP/O ratios. Methods Enzymol. 10, 41-47.

Ferrero, M. E., Ferrero, E., Gaja, G., and Bernelli-Zazzera, A. (1976). Adriamycin: Energy metabolism and mitochondrial oxidations in the heart of treated rabbits. Biochem. Pharmacol. 25, 125-130.

Feuerstein, G., Liu, G. L., Yue, T. L., Cheng, H. Y., Hieble, J. P., Arch, J. R., Ruffolo, R. R., Jr., and Ma, X. L. (1998). Comparison of metoprolol and carvedilol pharmacology and cardioprotection in rabbit ischemia and reperfusion model. Eur. J. Pharmacol. 351, 341-350.

Feuerstein, G. Z., Bril, A., and Ruffolo, R. R., Jr. (1997). Protective effects of carvedilol in the myocardium. Am. J. Cardiol. 80, 41L-45L.

Feuerstein, G. Z., Hamburger, S. A., Smith III, E. F., Bril, A., and Ruffolo, R. R., Jr. (1992). Myocardial protection with carvedilol. J Cardiovasc. Pharmacol. 19(Suppl. 1), S138-S141.

Foody, J. M., Farrell, M. H., and Krumholz, H. M. (2002). $\beta$-Blocker therapy in heart failure: Scientific review. JAMA 287, 883-889.

Gille, L., and Nohl, H. (1997). Analyses of the molecular mechanism of adriamycin-induced cardiotoxicity. Free Radical Biol. Med. 23, 775-782.

Henry, T. R., Solem, L. E., and Wallace, K. B. (1995). Channel-specific induction of the cyclosporine A-sensitive mitochondrial permeability transition by menadione. J. Toxicol. Environ. Health 45, 489-504.

Herman, E. H., Zhang, J., Chadwick, D. P., and Ferrans, V. J. (2000).
Comparison of the protective effects of amifostine and dexrazoxane against the toxicity of doxorubicin in spontaneously hypertensive rats. Cancer Chemother. Pharmacol. 45, 329-334.

Imondi, A. R. (1998). Preclinical models of cardiac protection and testing for effects of dexrazoxane on doxorubicin antitumor effects. Semin. Oncol. 25(Suppl. 10), 22-30.

Jagasia, D. H., and Shivkumar, K. (2002). Carvedilol reduced mortality and morbidity caused by myocardial infarction in patients with left ventricular dysfunction. ACP J. Club 136, 7.

Ji, L. L. and Mitchell, E. W. (1994). Effects of Adriamycin on heart mitochondrial function in rested and exercised rats. Biochem. Pharmacol. 47, 877-885.

Kamo, N., Muratsugu, M., Hongoh, R., and Kobatake, Y. (1979). Membrane potential of mitochondria measured with an electrode sensitive to tetraphenyl phosphonium and relationship between proton electrochemical potential and phosphorylation potential in steady state. J. Membr. Biol. 49, 105-121.

Kramer, J. H., and Weglicki, W. B. (1996). A hydroxylated analog of the beta-adrenoceptor antagonist, carvedilol, affords exceptional antioxidant protection to postischemic rat hearts. Free Radical Biol. Med. 21, 813-825.

Lahiri, A. (1996). Neurohumoral mechanisms in congestive heart failure and the role of drugs with multiple actions: A review of carvedilol. Am. J. Ther. 3, 237-247.

Lefrak, E. A., Pitha, J., Rosenheim, S., and Gottlieb, J. A. (1973). A clinicopathologic analysis of adriamycin cardiotoxicity. Cancer 32, 302-314.

Lysko, P. G., Webb, C. L., Gu, J. L., Ohlstein, E. H., Ruffolo, R. R., Jr., and Yue, T. L. (2000). A comparison of carvedilol and metoprolol antioxidant activities in vitro. J. Cardiovasc. Pharmacol. 36, 277-281.

Mailer, K., and Petering, D. H. (1976). Inhibition of oxidative phosphorylation in tumor cells and mitochondria by daunomycin and adriamycin. Biochem. Pharmacol. 25, 2085-2089.

Matsui, H., Morishima, I., Numaguchi, Y., Toki, Y., Okumura K., and Hayakawa, T. (1999). Protective effects of carvedilol against doxorubicininduced cardiomyopathy in rats. Life Sci. 65, 1265-1274.

Mimnaugh, E. G., Gram, T. E., and Trush, M. A. (1983). Stimulation of mouse heart and liver microsomal lipid peroxidation by anthracycline anticancer drugs: Characterization and effects of reactive oxygen scavengers. J. Pharmacol. Exp. Ther. 226, 806-816.

Mimnaugh, E. G., Trush, M. A., Bhatnagar, M., and Gram, T. E. (1985). Enhancement of reactive oxygen-dependent mitochondrial membrane lipid peroxidation by the anticancer drug adriamycin. Biochem. Pharmacol. 34, 847-856.

Nicolay, K., Fok, J. J., Voorhout, W., Post, J. A., and de Kruijff, B. (1986). Cytofluorescence detection of adriamycin-mitochondria interactions in isolated, perfused rat heart. Biochim. Biophys. Acta 887, 35-41.

Oliveira, P. J., Santos, D. J., and Moreno, A. J. (2000). Carvedilol inhibits the exogenous NADH dehydrogenase in rat heart mitochondria. Arch. Biochem. Biophys. 374, 279-285.

Olson, R. D., Boerth, R. C., Gerber, J. G., and Nies, A. S. (1981). Mechanism of adriamycin cardiotoxicity: Evidence for oxidative stress. Life Sci. 29, 1393-1401.

Olson, R. D., and Mushlin, P. S. (1990). Doxorubicin cardiotoxicity: Analysis of prevailing hypotheses [see comments]. FASEB J 4, 3076-3086.

Ponicke, K., Heinroth-Hoffmann, I., and Brodde, O. E. (2002). Differential effects of bucindolol and carvedilol on noradenaline-induced hypertrophic response in ventricular cardiomyocytes of adult rats. J. Pharmacol. Exp. Ther. 301, 71-76.

Powis, G. (1989). Free radical formation by antitumor quinones. Free Radical Biol. Med. 6, 63-101.

Praet, M., Calderon, P. B., Pollakis, G., Roberfroid, M., and Ruysschaert, J. M. 
(1988). A new class of free radical scavengers reducing adriamycin mitochondrial toxicity. Biochem. Pharmacol. 37, 4617-4622.

Praet, M., Pollakis, G., Goormaghtigh, E., and Ruysschaert, J. M. (1984). Damages of the mitochondrial membrane in Adriamycin treated mice. Cancer Lett. 25, 89-96.

Praet, M., and Ruysschaert, J. M. (1993). In-vivo and in-vitro mitochondrial membrane damages induced in mice by adriamycin and derivatives. Biochim. Biophys. Acta 1149, 79-85.

Ruffolo, R. R., Jr., Nichols, A. J., Stadel, J. M., and Hieble, J. P. (1991). Structure and function of alpha-adrenoceptors. Pharmacol. Rev. 43, 475-505.

Seifert, C. F., Nesser, M. E., and Thompson, D. F. (1994). Dexrazoxane in the prevention of doxorubicin-induced cardiotoxicity. Ann. Pharmacother. 28, 1063-1072.

Singal, P. K., Deally, C. M., and Weinberg, L. E. (1987). Subcellular effects of adriamycin in the heart: A concise review. J. Mol. Cell. Cardiol. 19, $817-828$.

Singal, P. K., and Iliskovic, N. (1998). Doxorubicin-induced cardiomyopathy [see comments]. N. Engl. J. Med. 339, 900-905.

Singal, P. K., Iliskovic, N., Li, T., and Kumar, D. (1997). Adriamycin cardiomyopathy: Pathophysiology and prevention. FASEB J 11, 931-936.

Singal, P. K., Li, T., Kumar, D., Danelisen, I., and Iliskovic, N. (2000). Adriamycin-induced heart failure: Mechanism and modulation. Mol. Cell. Biochem. 207, 77-86.

Singal, P. K., Siveski-Iliskovic, N., Hill, M., Thomas, T. P., and Li, T. (1995). Combination therapy with probucol prevents adriamycin-induced cardiomyopathy. J. Mol. Cell. Cardiol. 27, 1055-1063.

Siveski-Iliskovic, N., Hill, M., Chow, D. A., and Singal, P. K. (1995). Probucol protects against adriamycin cardiomyopathy without interfering with its antitumor effect. Circulation 91, 10-15.

Sokolove, P. M. (1988). Mitochondrial sulfhydryl group modification by adriamycin aglycones. FEBS Lett. 234, 199-202.

Solem, L. E., Heller, L. J., and Wallace, K. B. (1996). Dose-dependent increase in sensitivity to calcium-induced mitochondrial dysfunction and cardiomyocyte cell injury by doxorubicin. J. Mol. Cell. Cardiol. 28, 1023-1032.

Solem, L. E., Henry, T. R., and Wallace, K. B. (1994). Disruption of mitochondrial calcium homeostasis following chronic doxorubicin administration. Toxicol. Appl. Pharmacol. 129, 214-222.

Solem, L. E., and Wallace, K. B. (1993). Selective activation of the sodiumindependent, cyclosporin A-sensitive calcium pore of cardiac mitochondria by doxorubicin. Toxicol. Appl. Pharmacol. 121, 50-57.

Tadolini, B., and Franconi, F. (1998). Carvedilol inhibition of lipid peroxidation: A new antioxidative mechanism. Free Radical Res. 29, 377-387.

Taylor, R. W., Birch-Machin, M. A., Bartlett, K., and Turnbull, D. M. (1993). Succinatecytochrome $c$ reductase: Assessment of its value in the investiga- tion of defects of the respiratory chain. Biochim. Biophys. Acta 1181, 261-265.

Tepper, D. (2001). Frontiers in congestive heart failure: Dilated cardiomyopathy in dialysis patients-beneficial effects of carvedilol: A double-blind, placebo-controlled trial. Congest. Heart Fail. 7, 170.

Trounce, I. A., Kim, Y. L., Jun, A. S., and Wallace, D. C. (1996). Assessment of mitochondrial oxidative phosphorylation in patient muscle biopsies, lymphoblasts, and transmitochondrial cell lines. Methods Enzymol. 264, 484509 .

Wallace, D. C. (1992). Diseases of the mitochondrial DNA. Annu. Rev. Biochem. 61, 1175-1212.

Wallace, K. B. (2001). Doxorubicin-induced mitochondrial cardiomyopathy. In Mitochondria in Pathogenesis. (J. J. Lemasters and A.-L. Nieminen, Eds.), Chapt. 25, pp. 467-488. Kluwer Academic/Plenum Publishers, New York.

Wallace, K. B., Eells, J. T., Madeira, V. M., Cortopassi, G. and Jones, D. P. (1997). Mitochondria-mediated cell injury: Symposium overview. Fundam. Appl. Toxicol. 38, 23-37.

Yaoita, H., Sakabe, A., Maehara, K. and Maruyama, Y. (2002). Different effects of carvedilol, metoprolol, and propranolol on left ventricular remodeling after coronary stenosis or after permanent coronary occlusion in rats. Circulation 105, 975-980.

Yesair, D. W., Schwartzbach, E., Shuck, D., Denine, E. P., and Asbell, M. A. (1972). Comparative pharmacokinetics of daunomycin and adriamycin in several animal species. Cancer Res. 32, 1177-1183.

Yue, T. L., Cheng, H. Y., Lysko, P. G., McKenna, P. J., Feuerstein, R., Gu, J. L., Lysko, K. A., Davis, L. L., and Feuerstein, G. (1992a). Carvedilol, a new vasodilator and beta adrenoceptor antagonist, is an antioxidant and free radical scavenger. J. Pharmacol. Exp. Ther. 263, 92-98.

Yue, T. L., McKenna, P. J., Gu, J. L., Cheng, H. Y., Ruffolo, R. E., Jr., and Feuerstein, G. Z. (1994). Carvedilol, a new vasodilating beta adrenoceptor blocker antihypertensive drug, protects endothelial cells from damage initiated by xanthine-xanthine oxidase and neutrophils. Cardiovasc. Res. 28, 400-406.

Yue, T. L., McKenna, P. J., Ruffolo, R. R., Jr., and Feuerstein, G. (1992b). Carvedilol, a new beta-adrenoceptor antagonist and vasodilator antihypertensive drug, inhibits superoxide release from human neutrophils. Eur. J. Pharmacol. 214, 277-280.

Zhou, S., Heller, L. J., and Wallace, K. B. (2001). Interference with calciumdependent mitochondrial bioenergetics in cardiac myocytes isolated from doxorubicin-treated rats. Toxicol. Appl. Pharmacol. 175, 60-67.

Zhou, S., Starkov, A., Froberg, M. K., Leino, R. L., and Wallace, K. B. (2001). Cumulative and irreversible cardiac mitochondrial dysfunction induced by doxorubicin. Cancer Res. 61, 771-777. 